\title{
Combustion dynamic characteristics identification in a 9- point LDI combustor under choked outlet boundary conditions
}

\author{
Zhuohui J. He* and Clarence T. Chang ${ }^{\dagger}$ \\ NASA Glenn Research Center, Cleveland, Ohio, 44135
}

\begin{abstract}
Combustion dynamics data were collected at the NASA Glenn Research Center's CE-5 flame tube test facility under combustor outlet choked conditions. Two 9-point Swirl-Venturi Lean Direct Injection (SVLDI) configurations were tested in a rectangular cuboid combustor geometry. Combustion dynamic data were measured at different engine operational conditions up to inlet air pressure and temperature of 24.13 bar and $828 \mathrm{~K}$, respectively. In this study, the effects of acoustic cavity resonance, precessing vortex core (PVC), and non-uniform thermal expansion on the dynamic noise spectrum are identified by comparing the dynamic data that collected at various combustor inlet conditions along with combustor geometric calculations. The results show that the acoustic cavity resonance noises were seen in the counter-rotating pilot configuration but not in the co-rotating pilot configuration. Dynamic pressure noise band at around 0.9 $\mathrm{kHz}$ was only detected at the P'41 location (9.8 cm after fuel injector face) but not at the P'42 location (29 $\mathrm{cm}$ after the fuel injector face); the amplitude of this noise band depended on the thermal expansion ratio $\left(\mathrm{T}_{4} / \mathrm{T}_{3}\right)$. The noise band at around $1.8 \mathrm{kHz}$ was found to depend on the inlet air pressure or the air density inside the combustor. The PVC frequency was not observed in these two configurations.
\end{abstract}

\section{Introduction}

The aircraft certification process requires every new commercial aircraft design to meet a standard developed by the International Civil Aviation Organization (ICAO). This standard defines the maximum levels of $\mathrm{NO}_{\mathrm{x}}$ emissions and particulate matter for each commercial aircraft, and it is expected to be more restricted overtime. As a result, engine companies are focusing their future aircraft combustor designs on lean front end technologies, such as the GE Twin Annular Premixing Swirler (TAPS) concept and the P\&W Axially Controlled Stoichiometry (ACS) concept developed under NASA Environmental Responsibly Aviation (ERA) project [1]. NASA remains focused on Lean Direct Injection (LDI) as a candidate combustor concept for ultra-high pressure engine cycles, in which the combustor inlet temperature is high, the ignition delay time is short, and very little time exists to premix the fuel before combustion starts. In its extreme form, LDI injects fuel into the flame zone directly, mixing quickly as the fuel burns, reducing the probability and intensity of hot spots and keeping average flame temperature low.

Emission characteristics have been well studied for many LDI concepts $[2,10]$, but not combustion dynamics. A few studies have reported combustion dynamic on LDI concepts. One study had reported a second generation Woodard LDI dynamic data [4]. The study found combustion pressure fluctuations are low $(<3.5 \mathrm{kPa}$ peak to peak) for most conditions, except at one low power conditions, the peak-to-peak dynamic pressure fluctuation was greater than $10.0 \mathrm{kPa}$, and it is significantly depended on the fuel distribution ratio among the fuel-air mixers. Another study with a single mixer LDI injector found that instability increased with fuel air ratio, which might prevent full engine power operation [5].

Many sources could lead to combustion instability. Basic interactions and elementary processes are well described by Candel in a summary paper [6]. In brief, combustion dynamic is determined by combustor geometry, air flow dynamic, and heat release characteristic inside a combustor. For high amplitude instabilities to happen, pressure and heat release fluctuation need to be completely in phase to meet the Rayleigh criterions [7]. In this study, combustion dynamic data were collected at NASA Glenn Research Center's CE-5 flame tube test facility under outlet choked conditions. Two 9-point Swirl-Venturi Lean Direct Injection (SV-LDI) injector configurations were tested separately, and the combustor geometry was well defined as a rectangular cuboid $(7.62 \mathrm{~cm} \mathrm{X} 7.62 \mathrm{~cm} \mathrm{X}$ $45.72 \mathrm{~cm}$ ). Acoustic cavity resonance, precessing vortex core $(\mathrm{PVC})$, and non-uniform thermal expansion effects on the dynamic noise spectrum are identified by correlating the dynamic data with combustor operational conditions,

\footnotetext{
* Propulsion physicist, NASA Glenn Research Center

† System Engineer, NASA Glenn Research Center
} 
such as inlet air temperature, inlet air pressure, pressure drop across the injector face, and calculated adiabatic flame temperatures. The results from the two 9-point SV-LDI injector configurations and the effects of dynamic sampling locations are also addressed.

\section{Experimental facilities and hardware}

Experimental data used in this study were collected on Stand 2 of the CE-5 flame-tube test facility at NASA Glenn Research Center [8]. Figure 1 shows a picture of the combustion rig. Non-vitiated air was pre-heated to a maximum temperature of $830 \mathrm{~K}$ and maximum pressure of 24.13 bar. Commercial JP-8 aviation fuel was used. Fuel flow rates were measured by turbine meters and coriolis flow meters, and the air flow rate was measured by a venturi meter. The fuel-air mixture was injected into a flame-tube with a cast ceramic liner. A gas probe was placed $200 \mathrm{~mm}$ downstream of the injector to collect combustion products for analysis. The rest of the combustion products were cooled down to $500 \mathrm{~K}$ by mixing with sprayed water before exiting to an altitude exhaust system. The combusted gas samples were analyzed according to the standard gas-analysis procedure, SAE-ARP1256 [9].

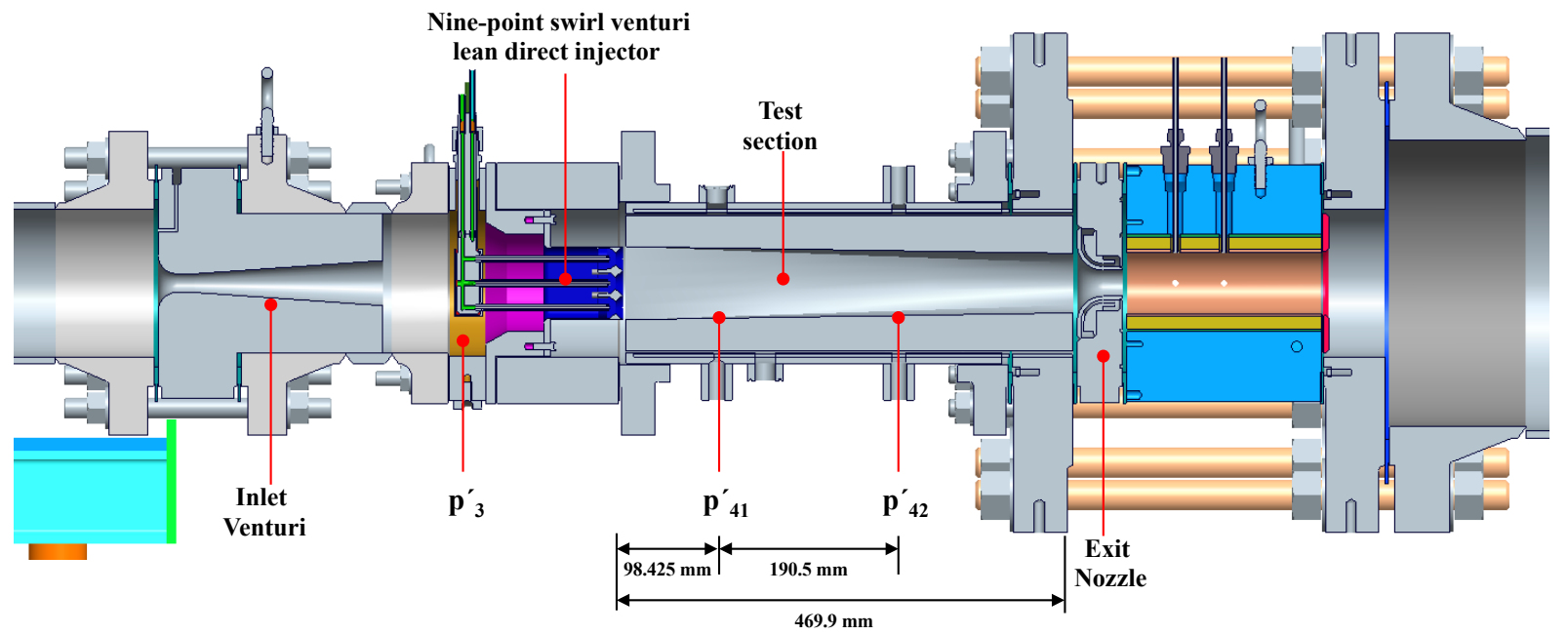

Figure 1: test rig and dynamic instrumentation location.

The SV-LDI module described here contains 9 identical fuel/air mixers in a $3 \times 3$ array as shown in Figure 2 . The 9 fuel/air mixers replace a single conventional fuel injector. Each fuel/air mixer consists of an air passage with a helical axial air swirler followed by a converging-diverging venturi section. A simplex fuel injector is inserted through the center of the air swirler; the fuel injector tip is at the venturi throat. Three fuel circuits are used in this test. One fuel circuit supplies fuel to the middle fuel air mixer, which acts as a pilot. The other two fuel circuits each feeds four surrounding fuel air mixers: one feeds the corners, and the other feeds directly to the fuel air mixers adjacent to the center fuel air mixer.

Two swirl configurations were tested. Figure $2 \mathrm{c}$ and $2 \mathrm{~d}$ show the differences between the two. The co-rotating pilot configuration had the pilot mixer swirler rotating at the same direction as the main mixers, while the counterrotating pilot configuration had the pilot mixer swirler rotating at the opposite direction. For this study, fuel air equivalence ratios are similar among the nine mixers.

Combustion chamber geometry was well defined as a rectangular cuboid $(7.6 \mathrm{~cm} \mathrm{X} 7.6 \mathrm{~cm} \mathrm{X} 46 \mathrm{~cm})$. Dynamic pressures were sampled from three locations axially. As indicated in Figure 1, the P' 3 was located upstream of the fuel injection, while the P' 41 was located $9.8 \mathrm{~cm}$ and the P' 42 was located $29 \mathrm{~cm}$ after the fuel injector face. Three tubes, $0.46 \mathrm{~cm}$ inside diameter, were inserted into the combustor at these three locations to collect combustion dynamic data. The sensors were placed $0.9 \mathrm{~m}$ away from the tube inlet, each with a $30 \mathrm{~m}$ tail to damp out the dynamic signals. A slow nitrogen purge flow at the end of the tail provided positive flow to keep the sensors from the hot combustor gas. The dynamic sensors used were piezoelectric, PCB Piezotronics, model 112A22. 


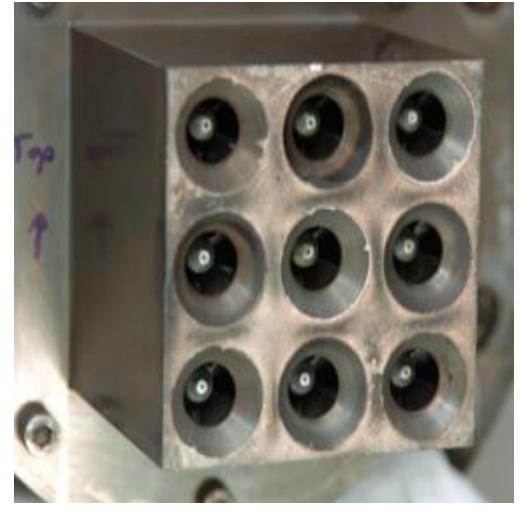

a) Injector hardware

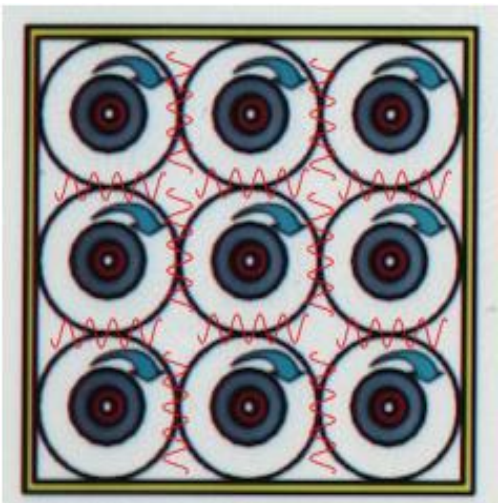

c) Co-rotating pilot configuration

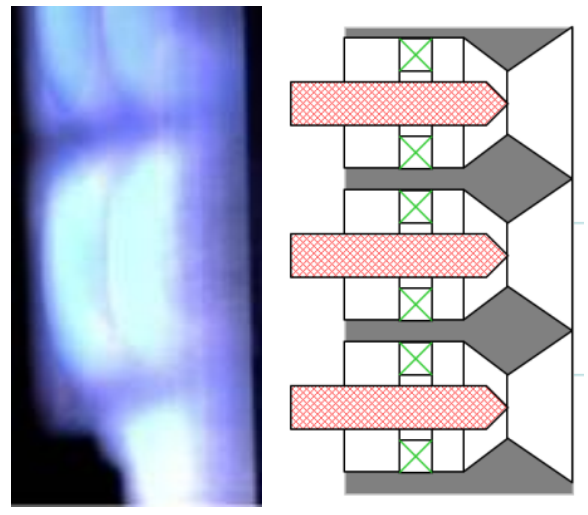

b) Cross-sectional flame image and drawing

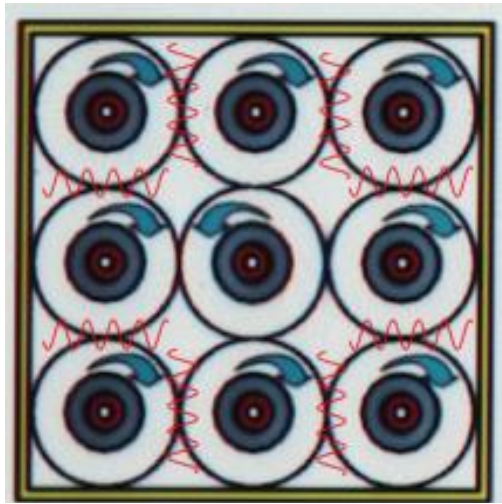

d) Counter-rotating pilot configuration

Figure 2: 9-point injector configuration a) injector hardware, b) cross-sectional drawing, c) with counter-rotating pilot, and d) with co-rotating pilot.

The dynamic pressure signals are transformed to frequency domain with using the Fast Fouier Transform (FFT) function in Matlab. Each dynamic pressure reading is recorded for 30 seconds at 20 or $30 \mathrm{kHz}$. For spectral processing, each recording is split into segments of $2^{15}$ points. As a result, each FFT used about 1.1 to 1.5 seconds of dynamic data. After the transformation, the frequency spectrum was corrected for line loss in the sampling line following Samuelson [10]. To reduce noise, the average values of these FFTs are reported in this study. The y-axis of the FFT plot is normalized by the combustor inlet air pressure, which is proportional to the square of the velocity fluctuations inside the combustor.

\section{Results and Discussion}

\section{Reacting Effects on Combustor Dynamics}

The dynamic pressure fluctuations under non-reacting conditions are significant up to $3.0 \mathrm{kHz}$ as shown in Figure 3. Unsteady flow or turbulence induces hydrodynamic pressure fluctuations [11]. The co-swirling rectangular $3 \times 3$ array layout introduces a powerful opposing-flow shear layer between every pair of injector elements. This feature provides rapid mixing in the combustor during high power operation when the latter may be operating under lean direct injection mode. While there is significant turbulent energy left through $3.0 \mathrm{kHz}$, the amplitude decays with increasing frequency. 
Combustion raises the noise spectrum amplitude across selected bands non-uniformly. The magnitude of the pressure fluctuation is much higher for the reacting condition than the non-reacting one. As shown in Figure 3, dynamic noises are significant up to about 6.0 to $7.0 \mathrm{kHz}$ under reacting conditions. Within the operational conditions that were tested in this study, large magnitude combustion instabilities were not been observed.

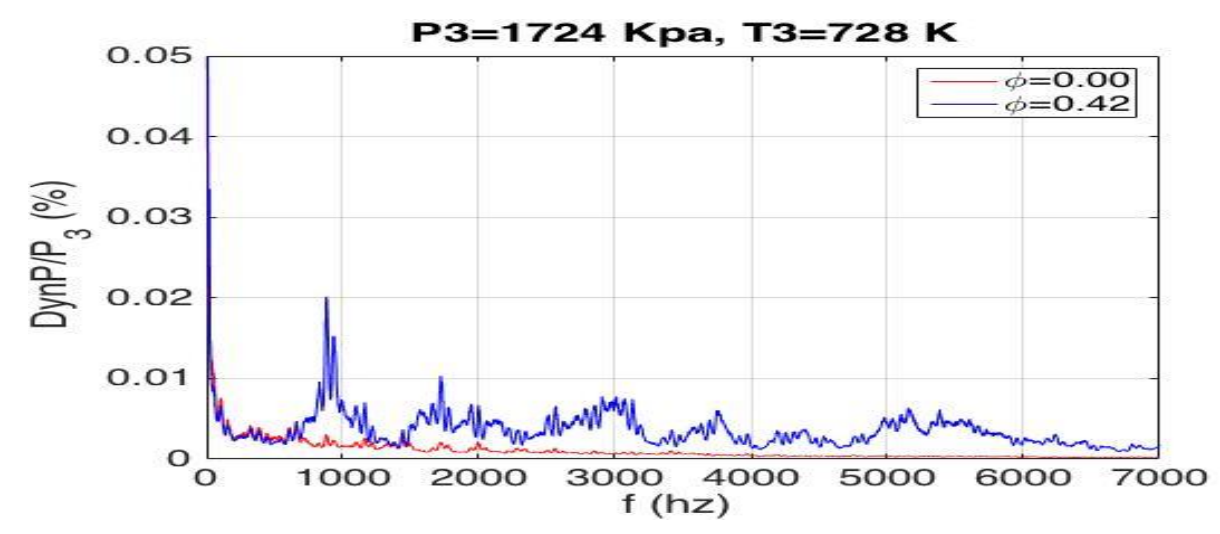

Figure 3: non-reacting vs. reacting dynamic pressure spectrums (FFT) at an inlet temperature of $727 \mathrm{~K}$ and inlet pressure of 17.2 bar (with co-rotating pilot configuration at the P'41 location)

\section{Flow Field Effects}

Reversing the pilot injector swirl direction removes the shear layer between it and its four adjacent neighbors by setting up cooperative flow at those borders, which reinforces the pilot flow. Figure $2 \mathrm{c}$ and $2 \mathrm{~d}$ show the drawings for the co-rotating pilot configuration and the counter-rotating pilot configuration. The red wavily lines represent noncooperative interactions between swirling air jets that generated by the fuel-air mixers. The dynamic spectrum is about the same for the counter-rotating and co-rotating pilot configurations for up to $3.0 \mathrm{kHz}$ (Figure 4). Two broadband noises are seen in the counter-rotating configuration spectrum at around $4.3 \mathrm{kHz}$ and $5.5 \mathrm{kHz}$, which are not seen in the co-rotating pilot configuration. In addition, the amplitude for noise at around $1.8 \mathrm{kHz}$ is significantly higher for the counter-rotating pilot configuration than for the co-rotating pilot configuration.

\section{Measurement Location Effects}

Dynamic pressures were taken at two locations axially inside the combustor. The P' 41 location is $10 \mathrm{~cm}$ downstream of the fuel injector face, and the P' 42 location is $20 \mathrm{~cm}$ further downstream. Figure $4 \mathrm{a}$ and $4 \mathrm{~d}$ compare the dynamic data that taken at these two locations for both configurations. The most significant difference in dynamic pressure fluctuations between these two locations is the presence of the $0.9 \mathrm{kHz}$ broadband noise at the P'41 location but not at the P'42 location.

There are two possible explanations. First, the $0.9 \mathrm{kHz}$ pressure fluctuation may have been damped before reaching the P'42 location due to viscous dissipation. Second, the $0.9 \mathrm{kHz}$ noise band could be near the node at the P'42 sampling location. With calculation, this noise band is corresponded the third harmonic mode of a resonance frequency under choked conditions, for which its wavelength is about $0.62 \mathrm{~m}$. A node is located at about $0.31 \mathrm{~m}$, which is near the P'42 location $(0.29 \mathrm{~m})$. As a result, no or low amplitude of the $0.9 \mathrm{kHz}$ noise band is seen at the $\mathrm{P}^{\prime} 42$ location. 

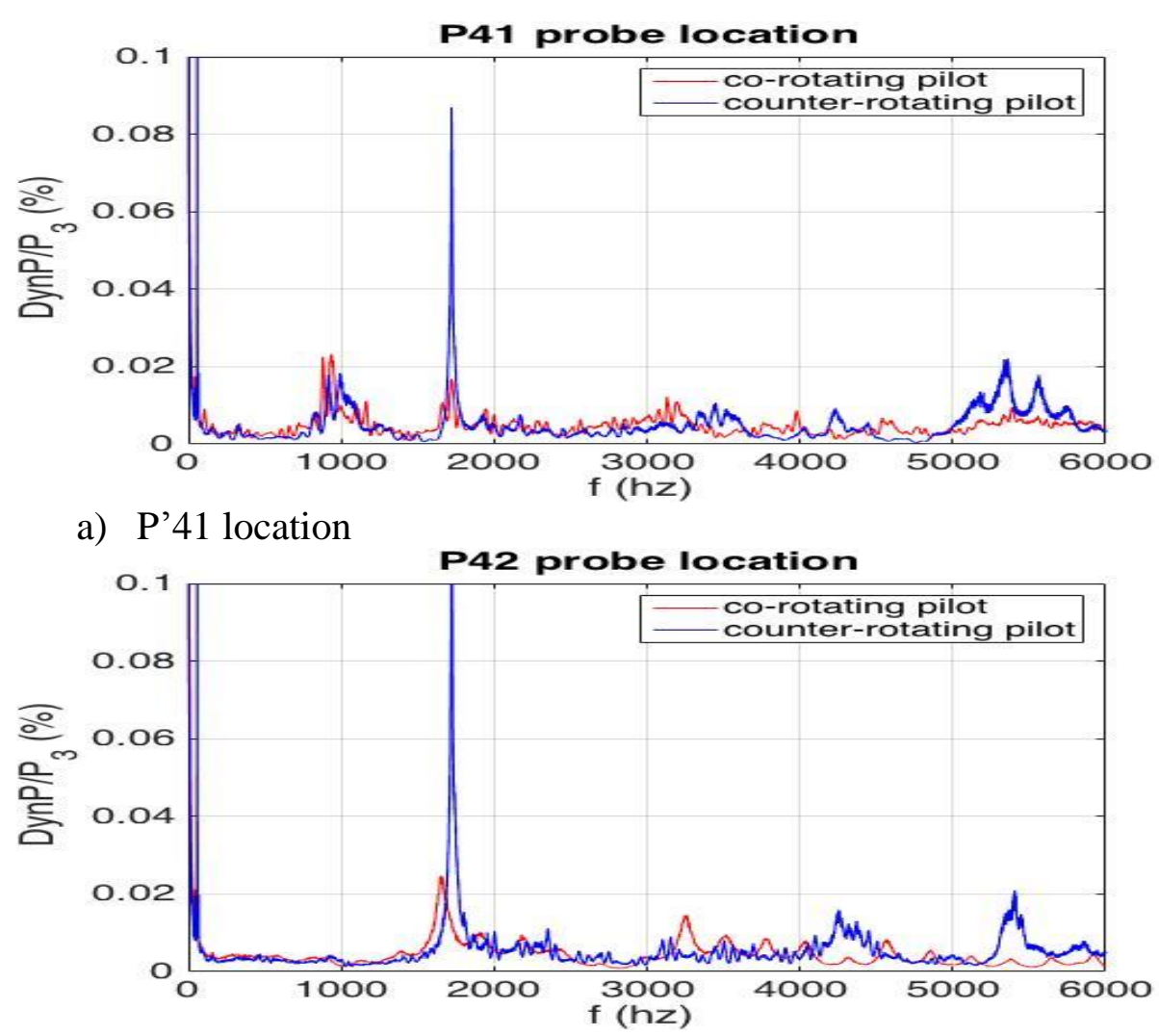

b) P'42 location

Figure 4: dynamic pressure spectrum (FFT) for counter-rotating pilot configuration and co-rotating pilot configuration at a) $\mathrm{P} 41$ and b) $\mathrm{P} 42$ location. $\left(\mathrm{T}_{3}=828 \mathrm{~K}, \mathrm{P}_{3}=24.1\right.$ bar, and $\left.\emptyset=0.47\right)$

\section{Non-uniform Thermal Expansion Effects}

Non-uniform fuel-air distribution can cause pressure perturbations through non-uniform localized thermal expansion that translates into non-uniform localized flow acceleration. Figure 5 contrasts the dynamic pressure spectrum for cases with varied thermal expansion ratios $\left(\mathrm{T}_{4} / \mathrm{T}_{3}\right)$ that ended with the same adiabatic flame temperatures $1866 \mathrm{~K}$ (Fig. 5a) and $1590 \mathrm{~K}$ (Fig. 5b). The amplitude of the $0.9 \mathrm{kHz}$ disturbance consistently varies with the thermal expansion ratio, regardless of variation in combustor inlet (Fig. 5) or outlet temperatures (Fig.6).

The amplitude of the dynamic noise band at around $1.8 \mathrm{kHz}$ did not show much variation as a function of the inlet air temperature, but it seems to depend on the inlet air pressure. Figure 7 shows the data taken at various equivalence ratio and at inlet air pressures of $10.3 \mathrm{bar}$ (7a), 17.2 bar (7b), and $24.1 \mathrm{bar}(7 \mathrm{c})$. These spectrums show that the amplitude of the $1.8 \mathrm{kHz}$ noise band increases as the inlet air pressure and equivalence ratio increase. The increase in equivalence ratio would lead to a larger thermal expansion. The inlet air pressure increases would yield an increase in air density but not the viscous damping force inside the combustor. As the results, the amplitude of this dynamic noise band increases as the inlet air pressure increases. 


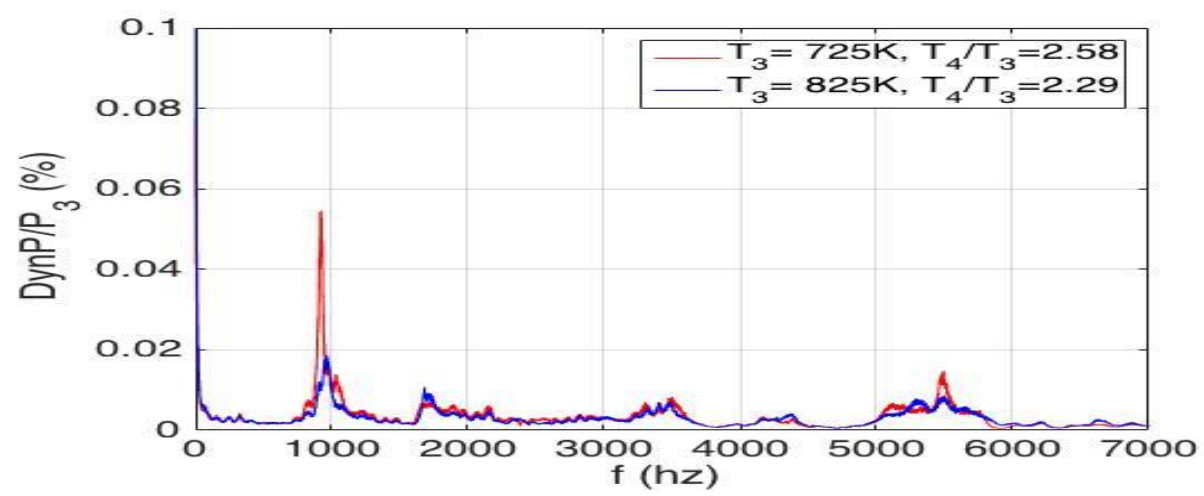

a) $\mathrm{T}_{4}=1866 \mathrm{~K}$

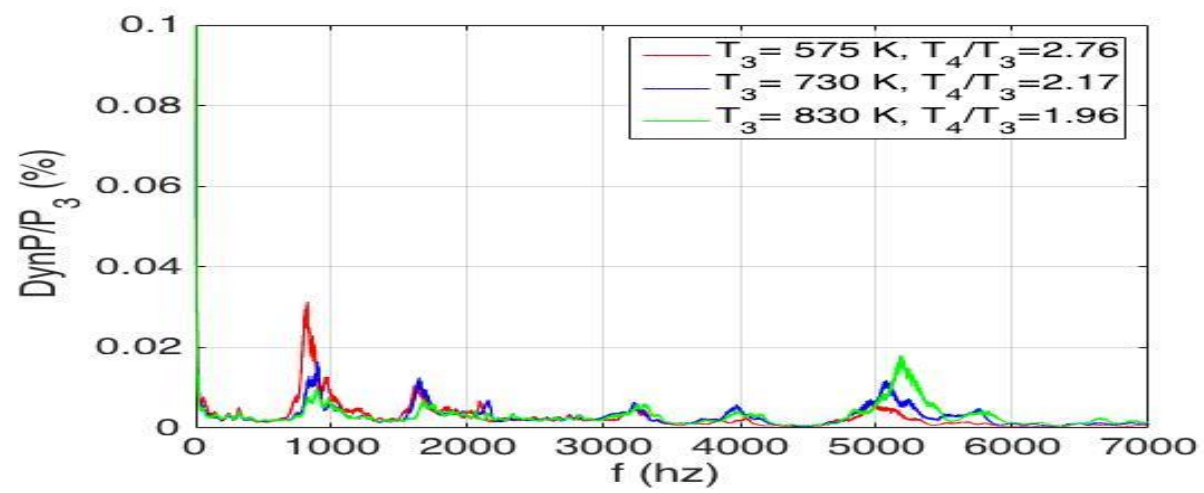

b) $\mathrm{T}_{4}=1590 \mathrm{~K}$

Figure 5: dynamic pressure spectrum (FFT) of the dynamic signals obtained at $\mathrm{P} 41$ location at various $\mathrm{T}_{3}$ conditions and at a) $\mathrm{T}_{4}=1866 \mathrm{~K}$, and b) $\mathrm{T}_{4}=1590 \mathrm{~K}\left(\mathrm{P}_{3}=10.3 \mathrm{bar}\right.$, with counter-rotating pilot configuration at the $\mathrm{P}^{\prime} 41$ location). 


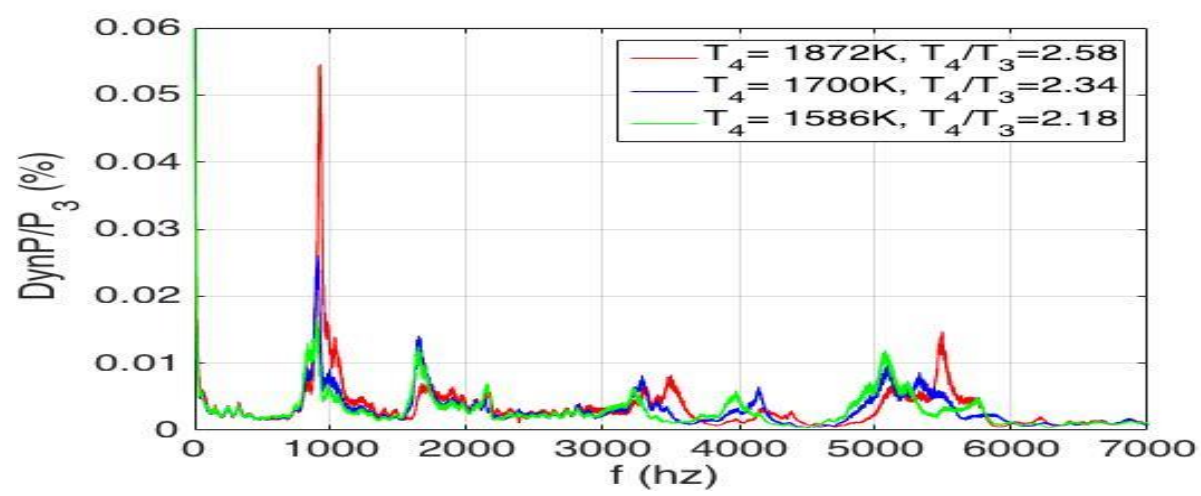

a) $\mathrm{T}_{3}=727 \mathrm{~K}$

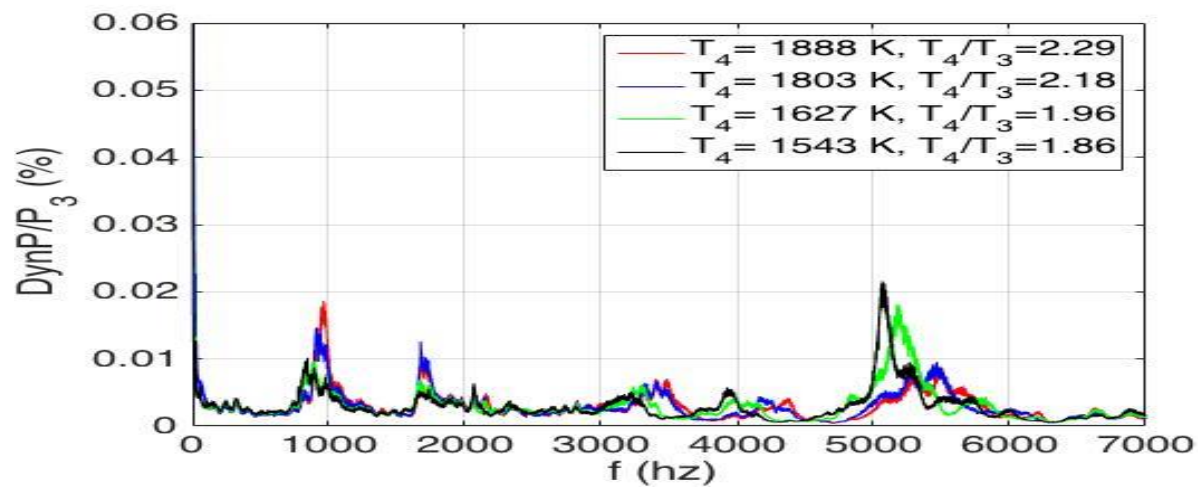

b) $\mathrm{T}_{3}=828 \mathrm{~K}$

Figure 6: dynamic pressure spectrum (FFT) of the dynamic signals obtained at $\mathrm{P} 41$ location at various $\mathrm{T}_{4}$ conditions and at a) $\mathrm{T}_{3}=727 \mathrm{~K}$, and b) $\mathrm{T}_{3}=828 \mathrm{~K}\left(\mathrm{P}_{3}=10.3 \mathrm{bar}\right.$, with counter-rotating pilot configuration at the $\mathrm{P}^{\prime} 41$ location) 


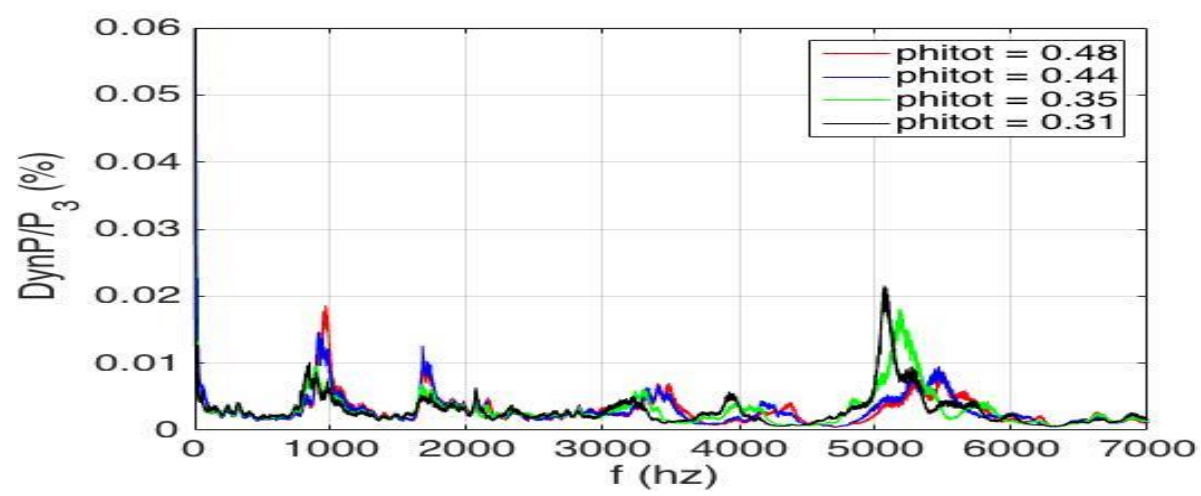

a) $\mathrm{P}_{3}=10.3 \mathrm{bar}$

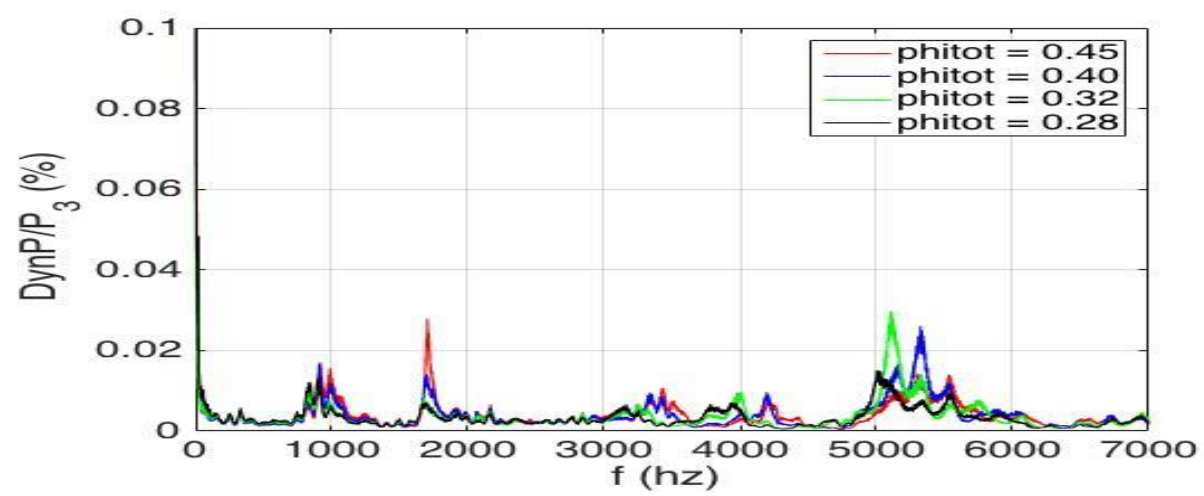

b) $\mathrm{P}_{3}=17.2 \mathrm{bar}$

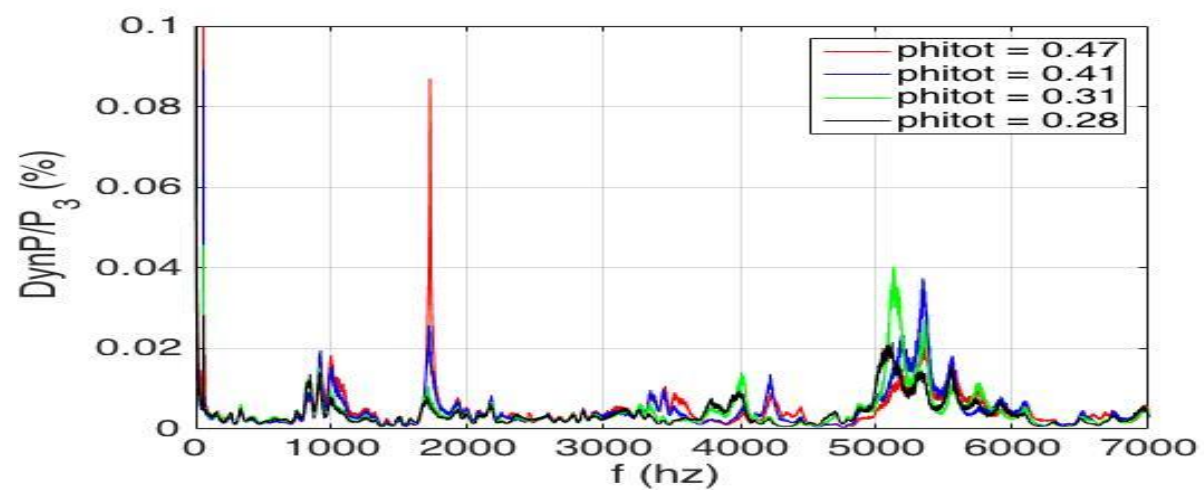

c) $\mathrm{P}_{3}=24.1 \mathrm{bar}$

Figure 7: dynamic pressure spectrum (FFT) of the dynamic signals obtained at $\mathrm{P} 41$ location at various fuel air equivalence ratio conditions and at a) $\mathrm{P}_{3}=10.3$ bar, b) $\mathrm{P}_{3}=17.2$ bar, c) $\mathrm{P}_{3}=24.1$ bar $\left(\mathrm{T}_{3}=828 \mathrm{~K}\right.$, with counterrotating pilot configuration at the $\mathrm{P}$ '41 location) 


\section{Acoustic Cavity Resonance Effects}

Figure 8 compares dynamic pressure spectrum (FFT) of the combustor dynamic data obtained at calculated adiabatic flame temperature $\left(\mathrm{T}_{4}\right)$ of $1810 \mathrm{~K}\left(\mathrm{P}_{3}=24.1\right.$ bar and $\left.\mathrm{T}_{3}=828 \mathrm{~K}\right)$ at two locations to the expected or calculated locations of the axial mode and transverse mode acoustic frequencies (not the amplitudes). Acoustic frequencies depend on combustor geometries and flame temperature, and it is a function of speed of sound and wavelength of the sound as shown as follow:

$$
\text { Equation 1: } \quad f=\frac{C}{\lambda}=\frac{\sqrt{\Upsilon R T_{4}}}{2 L / n}
$$

where $f$ is frequency, $c$ is speed of sound inside the combustor, which is function of flame temperature $\left(c=\sqrt{Y R T_{4}}\right)$, and wavelengths are dependent on the combustor geometries. The sixth order axial mode frequency and first order transverse mode looks to be over lapped under this experimental condition. The expected acoustic frequencies are located at around $0.9,1.8,2.7,3.6,4.5,5.4$ and $6.3 \mathrm{kHz}$.

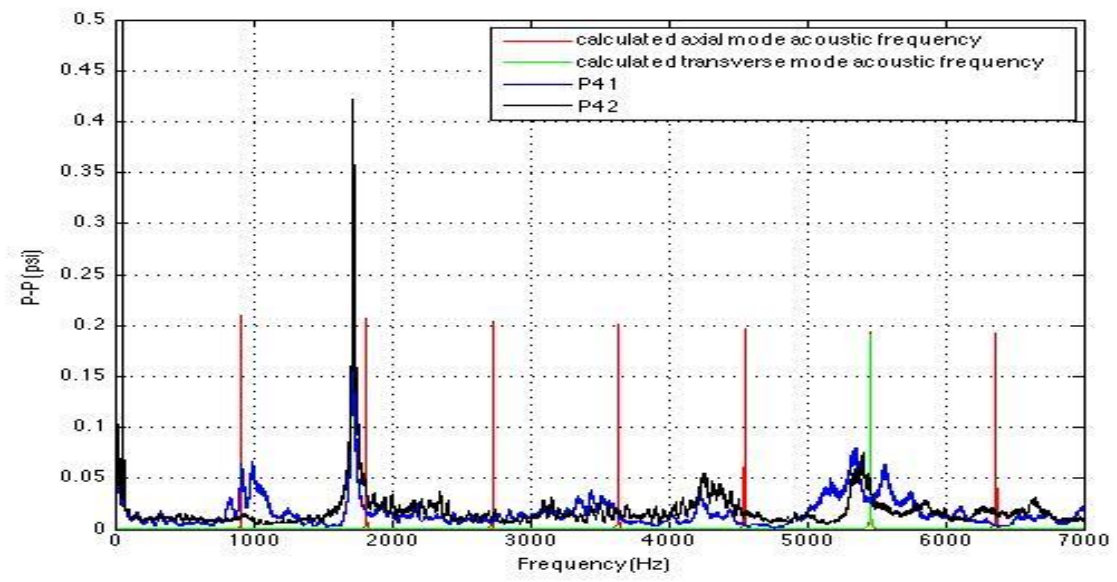

Figure 8: dynamic pressure spectrum (FFT) of the combustor dynamic data obtained at calculated adiabatic flame temperature $\left(\mathrm{T}_{4}\right)$ of $1810 \mathrm{~K}\left(\mathrm{P}_{3}=24.1\right.$ bar and $\left.\mathrm{T}_{3}=828 \mathrm{~K}\right)$ at two locations and the expected or calculated locations of the axial mode and transverse mode acoustic frequencies (with counter-rotating pilot configuration).

The flame temperature changes should cause the acoustic mode dynamic frequency to shifts accordingly, since the combustor geometry is fixed. Figure 9 shows the dynamic data obtained at the P' 42 location at different equivalence ratios for the counter-rotating pilot configuration. Shifts are seen at around $4.2 \mathrm{kHz}$ and $5.2 \mathrm{kHz}$ as the fuel air ratios changes. These frequencies might be corresponded to fifth, sixth harmonic axial acoustic modes and/or the first order transverse mode. As shown in Figure 8, the calculated acoustic frequencies are higher than the experimental obtained values. This may be because by that the flame tube (combustor chamber) is not completely adiabatic; the actual flame temperature is lower than the calculated $\mathrm{T}_{4}$. These acoustic frequencies shift only occurred with the counter-rotating pilot configuration but not the co-rotating pilot. The flow field differences between the two might be sources of these acoustic mode noises. 


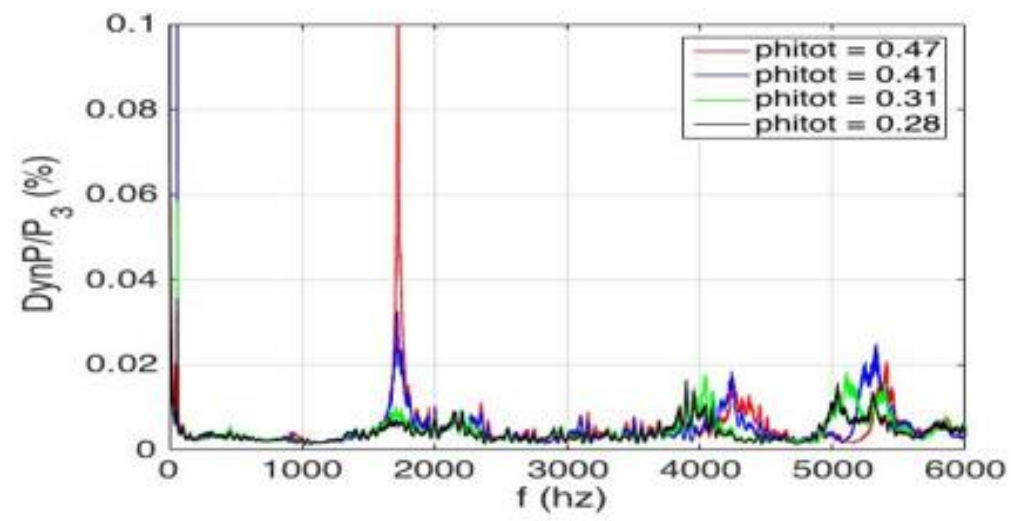

Figure 9: dynamic pressure spectrum (FFT) of the dynamic signals obtained at P42 location at $\mathrm{P}_{3}=24.1$ bar and $\mathrm{T}_{3}=828 \mathrm{~K}$, at various $\mathrm{T}_{4}$ conditions (with counter-rotating pilot configuration with counter-rotating pilot configuration at the P'42 location).

\section{Precessing Vortex Core Effects}

Precessing vortex core is a possible source of dynamic noise source. Each mixer has a 60-degree swirler, a swirl number of 1.0 [2]. The swirling flow generates a center recirculation zone for ignition stabilization and provides highly turbulence air near the fuel nozzle tip for fuel air mixing. This strong precessing vortex core can lead to a corresponding dynamic noise. This PVC frequency is close to the basic rotation rate of the air jet stream and increases linearly with flow rate [13]. In this study, differential air pressure drop conditions are examined and the resulting dynamic pressure spectrums are compared to identify the presence of a PVC induced frequency. Air pressure drop is linearly related to the square of the air flow velocity. As the result, the precessing vortex core (PVC) frequency is a function of the square root of the pressure drop over the fuel injector face. Figure 10 shows the dynamic pressure spectrum (FFT) at various pressure drop conditions $\left(\mathrm{T}_{3}=828 \mathrm{~K}, \mathrm{P}_{3}=11.3 \mathrm{bar}\right.$ ). With pressure drop decrease from $3.2 \%$ to $2.6 \%$ and to $2.1 \%$, the PVC frequencies should decrease by about $10 \%$ and $20 \%$ respectively. However, no shift by $10 \%$ or $20 \%$ is seen in this figure for any frequency. The amplitude for most pressure fluctuation is similar, except at around $1.8 \mathrm{kHz}$, which decreases as the pressure drop decreases.

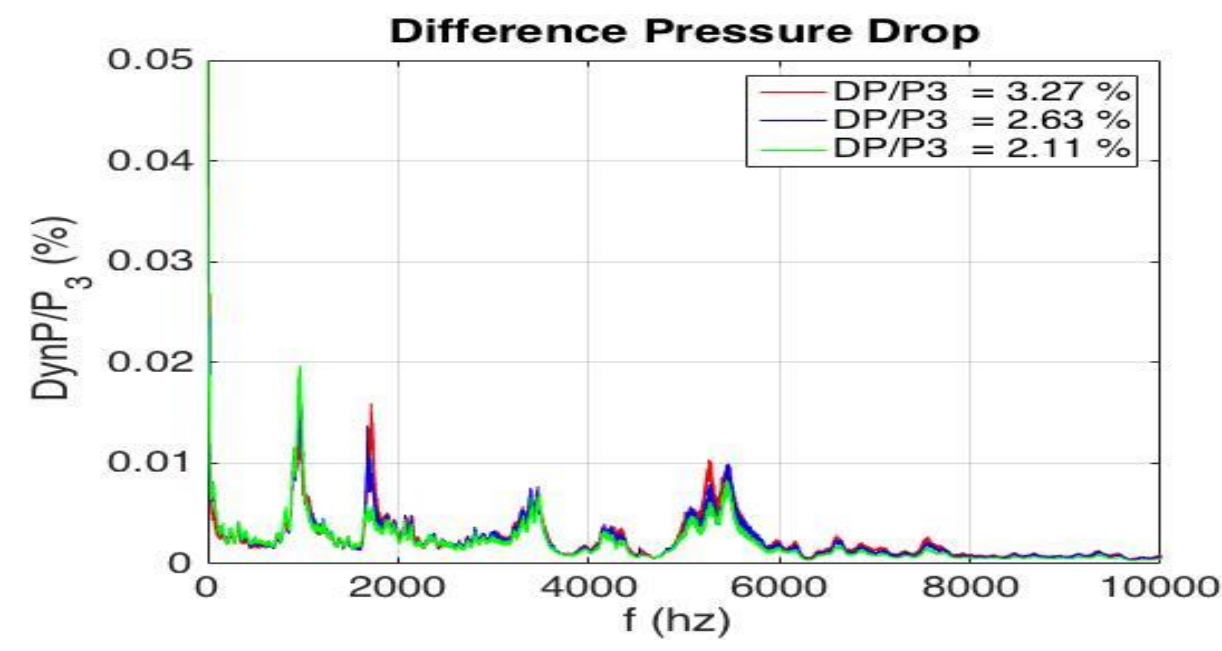

Figure 10: dynamic pressure spectrum (FFT) obtained at $\mathrm{P} 41$ location at $\mathrm{T}_{3}=828 \mathrm{~K}, \mathrm{P}_{3}=11.3$ bar, at various pressure drop conditions (with counter-rotating pilot configuration at the P'41 location). 


\section{Summary}

Dynamic pressure spectrums of two 9-point Swirl-Venturi Lean Direct Injection configurations are characterized in this study. Pressure fluctuation inside the combustor is air flow dynamic driven and enhanced by non-uniform heat release inside the combustor. The acoustic cavity resonance noises were seen in the counterrotating pilot configuration but not in the co-rotating pilot configuration. Dynamic pressure noise band at around 0.9 $\mathrm{kHz}$ was only detected at the P' 41 location $(9.8 \mathrm{~cm}$ after fuel injector face) but not at the P' 42 location ( $29 \mathrm{~cm}$ after the fuel injector face); the amplitude of this noise band depended on the thermal expansion ratio $\left(T_{4} / T_{3}\right)$. The noise band at around $1.8 \mathrm{kHz}$ was found to depend on the inlet air pressure or the air density inside the combustor. The $\mathrm{PVC}$ frequency was not observed in these two configurations.

\section{Acknowledgments}

This work supports the objectives and goals of NASA's Advanced Air Transportation Technology Project funded by the Aeronautics Research Mission Directorate 


\section{References}

[1] Chang, C., Lee, C., Kramer, \& Kramer, S. (2013). NASA Environmentally Responsible Aviation Project Develops Next_Generation Low-Emissions Combustor Technologies (Phase I). Aeronautics \& Aerospace Engineering, 2.

[2] Tacina, r., Lee, P., \& Wey, C. (2005). A Lean-Direct-Injection Combustor Using a 9 Point Swirl-Venturi Fuel Injector. ISABE, 1106.

[3] He, Z. J., Chang, C., \& Follen, C. (2014). NOx Emissions Performance and Correlation Equations for a Multipoint LDI Injector. NASA/TM, 218116.

[4] Tacina, K., Chang, C., Lee, P., \& Mongia, H. (2015). An assessment of combustion dynamics in a low-NOx, second-generation swirl-venturi lean direct injection combustion concept. ISABE, (p. 20249).

[5] DeLaat, J. C., \& Paxson, D. E. (2008). Characterization and Simulation of the Thermoacoustic Instability Behavior of an Advanced, Low Emissions Combustor Prototype. NASA/TM, 215291.

[6] Candel, S. (2002). Combustion Dynamics and Control: Progress and Challenges. Proceedings of the Combustion Institute, 29, pp. 1-28.

[7] Rayleigh, L. (1878). Nature, 18, 319-321.

[8] Bianco, J. (1995). NASA Lewis Research Center's combustor test facilities and capabilities. AIAA, (p. 2681). Candel, S. (2002). Combustion Dynamics and Control: Progress and Challenges. Proceedings of the Combustion Institute, 29, pp. 1-28.

[9] SAE International. (2011). Procedure for the Continuous Sampling and Measurement of Gaseous Emissions from Aircraft Turbine Engines. SAE ARP1256D, 2011-07

[10] Samuelson, R. (1969). Pneumatic Instrumentation Lines and their Use in Measuring Rocket Nozzle Pressure. Aerojet-General Corporation, Report No. NR-DR-0124.

[11] Williams, J. (1969). Hydrodynamic Noise. Annu Rev. Fluid Mech.(1), 197-222.

[12] Heath, C and Anderson R. et al. (2010) Optical Characterization of a Multipoint Lean Direct Injector for Gas Turbine Combustors: Velocity and Fuel Drop Size Measurements, NASA/TM-2010-216365.

[13] Moeck, J. P., Bourgouin, J.-F., Durox, D., Schuller, T., \& Candel, S. (2012). Nonlinear interaction between a precessing vortex core and acoustic oscillations in a turbulent swirling flame. Combustion and Flame, 159, 26502668. 